\title{
$3^{\circ} \bigcirc \supset \bigcirc \mid \begin{aligned} & \text { 3 simpósio paranaense de } \\ & \text { patologia das construções }\end{aligned}$
}

ISSN 2526-7248 artigo n. 3SPPC1011, pp. 116-129, 2018

\section{Influência do formato dos corpos de prova e da idade de cura nas leituras de velocidade de ultrassom, resistividade elétrica e resistência à compressão axial}

\author{
Godinho, Jayson Pereira'; Oliveira, Rafael Luiz Neves de ${ }^{2}$; Capraro, Ana Paula \\ Brandão ${ }^{3}$; Réus, Giovana Costa ${ }^{4}$; Medeiros, Marcelo Henrique Farias de ${ }^{5}$ \\ 1 Doutorando, Universidade Federal do Paraná, jayson.godinho.eng@gmail.com \\ ${ }^{2}$ Mestrando, Universidade Federal do Paraná, rafaell_neves@hotmail.com \\ ${ }^{3}$ Doutoranda, Universidade Federal do Paraná, anapcapraro@gmail.com \\ ${ }^{4}$ Doutoranda, Universidade Federal do Paraná, gio_reus@yahoo.com.br \\ ${ }^{5}$ Professor, Universidade Federal do Paraná, marcelo.ufpr@gmail.com
}

Resumo: As técnicas de ultrassom e de resistividade elétrica podem ser utilizadas como parâmetros para estimar a qualidade e a durabilidade do concreto. Diante disto, este trabalho avalia a influência do formato e das idades de cura dos corpos de prova nas leituras de velocidade de ultrassom, resistividade elétrica e resistência à compressão. Desse modo, foram moldados corpos de prova de concreto de três dimensões distintas, sendo elas: lajes armadas, prismas e cilindros. A resistência à compressão dos corpos de prova prismáticos foi $29 \%$ maior em relação aos corpos de prova cilíndricos, resultados estes que já eram esperados, visto que o fator de confinamento dos corpos de prova prismático é maior e resulta em uma maior restrição da prensa. Para os três formatos de corpos de prova, a velocidade de ultrassom aumentou ao longo do tempo e somente foi possível evidenciar a influência do formato e dimensões dos corpos de prova nas idades de 2 dias e 28 dias de cura. A resistividade elétrica para os três formatos de corpos de prova também aumentou ao longo do tempo, mas essa, foi influenciada diretamente pelo formato e dimensões, evidenciando que para corpos de prova com relação b/d (base do corpo de prova/distância equidistante entre eixos dos eletrodos do resistivímetro) menor que 6 , é necessário o cálculo de um fator de forma.

Palavras-chave: Fator de forma, Ensaios não-destrutivos, Concreto armado, Durabilidade do concreto.

\begin{abstract}
Ultrasonic and electrical resistivity techniques can be used as parameters to estimate the quality and durability of the concrete. In view of this, this work evaluates the influence of the format and the curing ages of the specimens in the ultrasonic velocity, electrical resistivity and compressive strength readings. In this way, concrete specimens of three different dimensions were molded, being: reinforced slabs, prisms and cylinders. The compressive strength of the prismatic specimens was $29 \%$ larger than the cylindrical test specimens, which were already expected, since the confinement factor of the prismatic specimens is larger and results in a greater restriction of the press. For the three formats of specimens, the ultrasound velocity increased over time and it was only possible to evidence the influence of the shape and dimensions of the specimens at the ages of 2 days and 28 days of cure. The electrical resistivity for the three test specimens also increased over time, but this was influenced directly by the shape and dimensions, showing that for test specimens with $\mathrm{b} / \mathrm{d}$ ratio (test specimen base / distance equidistant between axis of the resistivity meter) less than 6 , a shape factor is required.
\end{abstract}

Keywords: Form factor, Non-destructive testing, Reinforced concrete, Durability of concrete. 
GODINHO, J.P. et al., INFLUÊNCIA DO FORMATO DOS CORPOS DE PROVA E DA IDADE DE CURA NAS LEITURAS DE VELOCIDADE DE ULTRASSOM, RESISTIVIDADE ELÉTRICA E RESISTÊNCIA À COMPRESSÃO AXIAL. $3^{\circ}$ Simpósio Paranaense de Patologia das Construções (30 SPPC), artigo 3SPPC1011, pp. 116 - 129. 2018. DOI: 10.4322/2526-7248.011

\section{Introdução}

A durabilidade de uma estrutura de concreto pode ser entendida como a manutenção da sua funcionalidade em serviço, com a segurança esperada, durante um período especificado em projeto [1]. Na fase de dimensionamento da estrutura, as questões de durabilidade têm sido levadas cada vez mais em conta, pelos elevados custos de reparação, cujas causas decorrem de falhas nos materiais, execução e projetos [2].

A porosidade do concreto é um fator de grande impacto na manutenção da sua qualidade e durabilidade, estando diretamente relacionada ao fluxo de fluidos agressivos para o interior do mesmo $[2,3]$.

Ao longo da hidratação do cimento os produtos formados passam a ocupar gradativamente os espaços nos quais existiam água e ar e, assim, ocorre a redução do tamanho e da quantidade dos vazios na pasta de cimento, a qual, mesmo com essa redução, apresenta porosidade maior do que os agregados [2].

Monfore [4] afirma que a água evaporável presente nos poros capilares do concreto apresenta íons de cálcio, sulfatos, hidroxilas, além de cátions de sódio e potássio que garantem que a condução de eletricidade no interior do concreto ocorra de forma eletrolítica. Durante o endurecimento do concreto, tanto a água livre na mistura, como os íons dissolvidos nela influenciam na variação das propriedades elétricas neste meio [5].

Estudos de Whittington, Mccarter e Forde [6] mostraram que a resistividade elétrica dos agregados é muito superior à resistividade da pasta de cimento e, por consequência disso, a passagem de corrente elétrica ocorreria essencialmente pela última. Progredindo a hidratação, apesar de a dissolução iônica prover uma facilitação para a passagem de corrente elétrica, isso seria compensado pela redução no volume dos poros existentes, o que dificultaria a movimentação desses íons, situação aliada ao fato de o caminho da transmissão paulatinamente ficar menos linear, por conta do aumento da tortuosidade entre os poros por onde transita o fluxo, ou seja, o caminho percorrido pela corrente se torna maior [7].

Nesse sentido, cabe salientar que o grau de saturação do concreto é fator preponderante para a resistividade. Neville [3] mostrou que o concreto úmido representa um eletrólito com resistividade em torno de $10^{2} \mathrm{ohm} . \mathrm{m}$. Já em concretos secos em estufa, esse parâmetro pode sofrer uma elevação da ordem de $10^{7}$, podendo ser classificado como material isolante. Assim, para que a umidade não interfira diretamente nas medições, pode-se adotar como referência a medição das propriedades elétricas do concreto em material totalmente saturado sendo que, nesse caso, a resistividade elétrica tende a se tornar uma medida indireta da conectividade entre os poros do material [8].

A técnica de ultrassom, assim como a de resistividade elétrica pode ser utilizada como parâmetro para estimar a qualidade e a durabilidade do concreto. Este método consiste, basicamente, na medição do tempo necessário para que um pulso de ondas ultrassônicas atravesse um espaço entre dois transdutores eletrônicos [9]. 
Ao posicionar esses receptores em faces diretamente opostas de um corpo de prova, a medida linear deste elemento é utilizada para o cálculo da velocidade de propagação do pulso, que pode ser usado como indicador da homogeneidade.

Desta forma, quanto maior é a velocidade de propagação de onda ultrassônica, maior é a densidade do concreto e consequentemente, maior é o refinamento dos poros e sua durabilidade frente a penetração de agentes agressivos [10].

De acordo com Ribeiro et al. [11] e NBR 8802 [9], apesar de não existir uma relação direta entre a velocidade da onda no concreto e sua resistência mecânica, 0 conceito de que a onda sonora se propaga mais rapidamente no meio mais denso indica a possibilidade de se correlacionar a velocidade medida com a qualidade do concreto executado, podendo indicar um concreto de qualidade muito fraca, com velocidade de onda ultrassônica $<2.000 \mathrm{~m} / \mathrm{s}$, até um material excelente com velocidade de onda superior a $4.500 \mathrm{~m} / \mathrm{s}$.

Deste modo, o objetivo do presente trabalho é avaliar a influência do formato e das idades de cura dos corpos de prova na variação das leituras de velocidade de ultrassom, resistividade elétrica e resistência à compressão axial.

\section{Programa experimental}

Foram moldados 3 formatos diferentes de corpos de prova de concreto, sendo eles: a) 5 cilíndricos, de dimensões $10 \mathrm{~cm} \times 20 \mathrm{~cm}$; b) 10 prismáticos de dimensões $4 \mathrm{~cm}$ x $4 \mathrm{~cm} \times 16 \mathrm{~cm} \mathrm{e} \mathrm{c)} 10$ lajes armadas, de dimensões $25 \mathrm{~cm} \times 25 \mathrm{~cm} \times 7 \mathrm{~cm}$ (Figura 1).

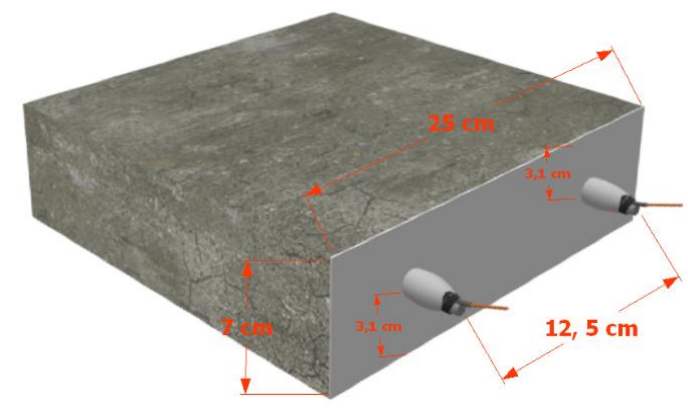

Figura 1: Esquema da laje armada moldada para o presente estudo

s lajes armadas foram moldadas com duas barras de aço carbono CA 50, de $8 \mathrm{~mm}$ de diâmetro, $30 \mathrm{~cm}$ de comprimento e de $3 \mathrm{~cm}$ de cobrimento, atendendo aos requisitos da NBR 6118 [12].

Todos os corpos de prova foram mantidos em cura por imersão, em água saturada com cal, com temperatura ambiente durante todo o período de 120 dias do experimento, de acordo com a NBR 5738 [13].

\subsection{Materiais utilizados}

O cimento utilizado no experimento foi o CP V-ARI, adotado por este apresentar menor teor de adições (90\% a 95\% de clínquer), adições estas que retardam as 
GODINHO, J.P. et al., INFLUÊNCIA DO FORMATO DOS CORPOS DE PROVA E DA IDADE DE CURA NAS LEITURAS DE VELOCIDADE DE ULTRASSOM, RESISTIVIDADE ELÉTRICA E RESISTÊNCIA À COMPRESSÃO AXIAL. $3^{\circ}$ Simpósio Paranaense de Patologia das Construções (30 SPPC), artigo 3SPPC1011, pp. 116 - 129. 2018. DOI: 10.4322/2526-7248.011

reações de hidratação do concreto e seriam um parâmetro de influência nos ensaios realizados no presente estudo. As propriedades físicas e mecânicas do cimento são informadas na Tabela 1 e a composição química é informada na Tabela 2.

Tabela 1: Propriedades físicas e mecânicas do cimento CP V-ARI.

Fonte: Fabricante

\begin{tabular}{cccccccc}
\hline \multicolumn{2}{c}{ Ensaios Físicos } & \multicolumn{2}{c}{ Tempo de pega } & \multicolumn{3}{c}{ Resistência à compressão (MPa) } \\
\hline Blaine $\left(\mathrm{cm}^{2} / \mathrm{g}\right)$ & $\# 200(\%)$ & Início (min.) & Fim (min.) & 1 dias & 3 dias & 7 dias & 28 dias \\
\hline 4710 & 0,3 & 250 & 310 & 22,2 & 37,1 & 41,5 & 49,2 \\
\hline
\end{tabular}

Tabela 2: Composição química do cimento CP V-ARI.

Fonte: Fabricante

\begin{tabular}{|c|c|c|c|c|c|c|c|c|c|}
\hline \multicolumn{9}{|c|}{ Parâmetros químicos (\%) } & \multirow{3}{*}{$\begin{array}{c}\begin{array}{l}\text { Perda ao } \\
\text { fogo }(\%)\end{array} \\
3.5\end{array}$} \\
\hline $\mathrm{CaO}$ & $\mathrm{SiO}_{2}$ & $\mathrm{Al}_{2} \mathrm{O}_{3}$ & $\mathrm{SO}_{3}$ & $\mathrm{Fe}_{2} \mathrm{O}_{3}$ & $\mathrm{MgO}$ & $\mathrm{K}_{2} \mathrm{O}$ & $\mathrm{TiO}_{2}$ & $\mathrm{Na}_{2} \mathrm{O}_{6}$ & \\
\hline 62,75 & 19,2 & 4,19 & 3,04 & 2,85 & 2,54 & 1,68 & 0,17 & 0,05 & \\
\hline
\end{tabular}

A caracterização dos agregados seguiu as recomendações das normas NM 248 [14], NM 52 [15] e NM 53 [16]. O agregado miúdo, oriundo da região metropolitana de Curitiba, foi classificado como areia fina natural, com distribuição granulométrica na zona ótima. O agregado graúdo, também oriundo da região metropolitana de Curitiba, de origem basáltica, foi caracterizado como brita 0 , com diâmetro máximo característico (DMC) de 12,5 $\mathrm{mm}$. A massa específica do agregado miúdo foi de $2,62 \mathrm{~g} / \mathrm{cm}^{3}$ e do agregado graúdo foi de $2,88 \mathrm{~g} / \mathrm{cm}^{3}$.

\subsection{Dosagem do concreto}

O traço adotado como referência foi de 1:3,2:3,7 (cimento, areia, brita) e a relação água/cimento de 0,59, abatimento do tronco de cone de $160 \pm 10 \mathrm{~mm}$, com a utilização de aditivo superplastificante à base de policarboxilato, sendo estes parâmetros adaptados de um traço comercializado em uma usina de concreto da região de Curitiba.

O adensamento foi realizado manualmente, com haste metálica padronizada, sendo o preenchimento dos corpos de prova feito em 2 camadas de concreto, para todas as amostras, como preconizado pela NBR 5738 [13].

\subsection{Ensaios de resistência à compressão axial e à tração na flexão}

A resistência à compressão axial foi determinada utilizando corpos de prova cilíndricos de dimensões $10 \mathrm{~cm} \times 20 \mathrm{~cm}$, conforme a norma NBR 7215 [17], com somente 28 dias de cura submersa.

Com o objetivo de avaliar o efeito da forma dos corpos de prova nos ensaios mecânicos do concreto, também foram moldados corpos de prova prismáticos de dimensões $4 \mathrm{~cm} \times 4 \mathrm{~cm} \times 16 \mathrm{~cm}$ e realizados ensaios de resistência à tração na flexão e à compressão axial, aos 28 dias e 100 dias de cura submersa, utilizando como base a norma NBR 13279 [18]. 


\subsection{Velocidade de ultrassom e resistividade elétrica}

Foram realizados os ensaios de ultrassom em todos os corpos de prova conforme a NBR 8802 [9], para verificar a variação da velocidade de propagação de ondas no interior dos corpos de prova nas idades de 2 dias, 16 dias, 28 dias, 41 dias, 55 dias e 100 dias. Foi utilizado um aparelho digital de ultrassom modelo Pundit Lab. da marca Proceq (Figura 2a), gel de ultrassonografia para o contato dos transdutores, sendo eles de $54 \mathrm{kHz}$ para os corpos de prova cilíndricos e para as lajes, e de 250 $\mathrm{kHz}$ para os corpos de prova prismáticos, sendo que as leituras foram realizadas de forma direta (Figura 2b), atendendo aos requisitos do fabricante.

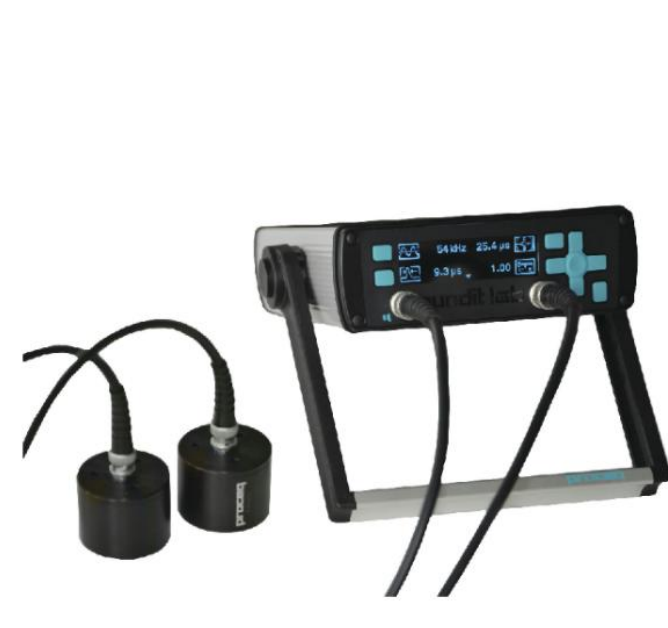

a)

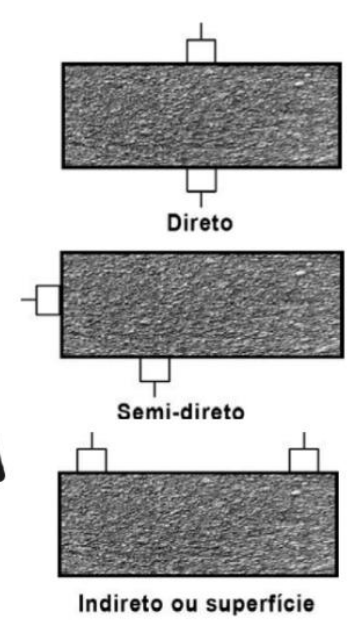

b)

Figura 2: Aparelhagem digital de medição da velocidade de ultrassom no concreto. a) equipamento Pundit Lab.; b) disposições dos transdutores comumente utilizadas. Fonte: Adaptado de Proceq [19]

De acordo com a anexo A da NBR 8802 [9] que trata sobre a verificação da homogeneidade do concreto, é necessário evitar os pontos onde exista grande concentração de armadura, principalmente no sentido longitudinal ao de propagação das ondas ultrassônicas, pois a velocidade de propagação de ondas é maior no aço do que no concreto. E para atender a esse requisito, as leituras foram executas transversalmente à direção das duas barras de aço do interior das lajes armadas.

O ensaio de resistividade elétrica foi realizado conforme a norma UNE 83988-2 [20] também nas idades de 2 dias, 16 dias, 28 dias, 41 dias, 55 dias e 100 dias. Utilizouse um resistivímetro modelo Resipod da marca Proceq (Figura 3a) que segue o princípio do método Wenner, também conhecido como método dos quatro eletrodos, sendo também necessário evitar os locais do concreto com armaduras, pois a presença do aço fazem com que as leituras de resistividade sejam subestimadas. Este é um ensaio não destrutivo que mensura a resistividade elétrica próxima à superfície e é realizado encostando-se a extremidade de quatro eletrodos equidistantes e alinhados sobre a superfície do concreto [21]. Durante o ensaio é emitida uma corrente alternada com frequência entre 25 e $150 \mathrm{~Hz}$ e tensão entre 6 e $12 \mathrm{~V}$ a partir dos dois eletrodos externos. A diferença de potencial é obtida por meio 
GODINHO, J.P. et al., INFLUÊNCIA DO FORMATO DOS CORPOS DE PROVA E DA IDADE DE CURA NAS LEITURAS DE VELOCIDADE DE ULTRASSOM, RESISTIVIDADE ELÉTRICA E RESISTÊNCIA À COMPRESSÃO AXIAL. $3^{\circ}$ Simpósio Paranaense de Patologia das Construções (30 SPPC), artigo 3SPPC1011, pp. 116 - 129. 2018. DOI: 10.4322/2526-7248.011

dos eletrodos internos, o que possibilita o cálculo da resistividade elétrica utilizando a Lei de Ohm [11].

Para os corpos cilíndricos de dimensões $10 \mathrm{~cm} \times 20 \mathrm{~cm}$, as medições foram feitas com eletrodos posicionados a uma distância equidistante (d) de $5 \mathrm{~cm}$ uns dos outros e foi aplicado um fator de forma de 0,377, que é estabelecido pela norma UNE 83988-2 [20], como meio de converter os dados obtidos no corpo de prova cilíndrico $10 \mathrm{~cm}$ x $20 \mathrm{~cm}$ para uma medida ideal obtida em meio semi-infinito, grande 0 suficiente para que as linhas equipotenciais e linhas de fluxo de corrente não saiam do corpo de prova e se fechem, não alterando os valores da resistividade elétrica obtido nas leituras com o equipamento (Figura 3b).
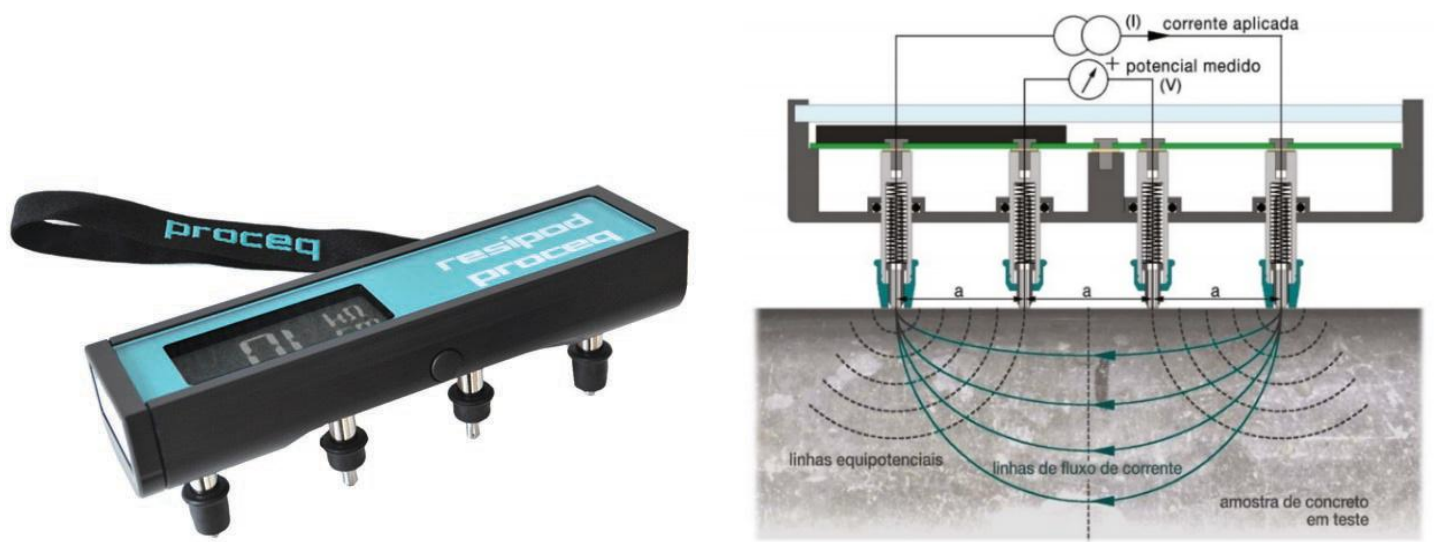

Figura 3: Aparelhagem para a medição da resistividade elétrica do concreto. a) Resipod. b) princípio de medição pelo método de Wenner.

Fonte: Adaptado de Proceq [22]

Para os corpos de prova prismáticos de dimensões $4 \mathrm{~cm} \times 4 \mathrm{~cm} \times 16 \mathrm{~cm}$, as medições foram realizadas por meio de um suporte com eletrodos que possuem espaçamento variável. Deste modo foi possível posicionar os eletrodos a uma distância equidistante (d) de $3,5 \mathrm{~cm}$ uns dos outros e por virtude desse espaçamento e da dimensão dos corpos de prova, foi aplicado um fator de forma de 0,172 conforme indica a norma UNE 83988-2 [20]. Para as lajes armadas, as leituras também foram realizadas com os eletrodos a uma distância equidistante (d) de $3,5 \mathrm{~cm}$ uns dos outros.

$\mathrm{Na}$ Figura 4 é apresentado o efeito da geometria e dimensões de peças de concreto prismáticos na determinação da resistividade elétrica pelo método dos quatro eletrodos de Wenner.

A base (b) das lajes armadas possui $25 \mathrm{~cm}$ e o espaçamento entre os eixos (d) adotado nas leituras foi de $3,5 \mathrm{~cm}$, resultando assim em uma relação $\mathrm{h} / \mathrm{d}(7 / 3,5)$ igual a 2 e em uma relação b/d $(25 / 3,5)$ igual a $7,142 \approx 7$ e de acordo com Gowers e Millard [23], corpos de prova com relação b/d (base do corpo de prova/distância equidistante entre eixos dos eletrodos do resistivímetro) maiores que 6 , possuem uma relação resistividade aparente (medida)/resistividade real próxima de 1 (Figura 4), não sendo necessário aplicar um fator de forma, uma vez que podem ser considerados como meios semi-infinitos, como é o caso das lajes armadas. 
Para comprovar a eficácia do gráfico de Gowers e Millard [23] apresentado na Figura 4 para estimar os fatores de forma de corpos de prova prismáticos, foi calculado esse fator para os corpos de prova de dimensões $4 \mathrm{~cm} \times 4 \mathrm{~cm} \times 16 \mathrm{~cm}$. A relação $\mathrm{h} / \mathrm{d}$ é igual a $1,142 \approx 1$ e a relação b/d é igual a $1,142 \approx 1$, fazendo com que a relação resistividade aparente (medida)/resistividade real seja $\leq 6$, gerando um fator de forma de $1 / 6=0,166$, bem próximo do valor 0,172 definido pela norma UNE 83988-2 [20], com apenas 4\% de diferença.

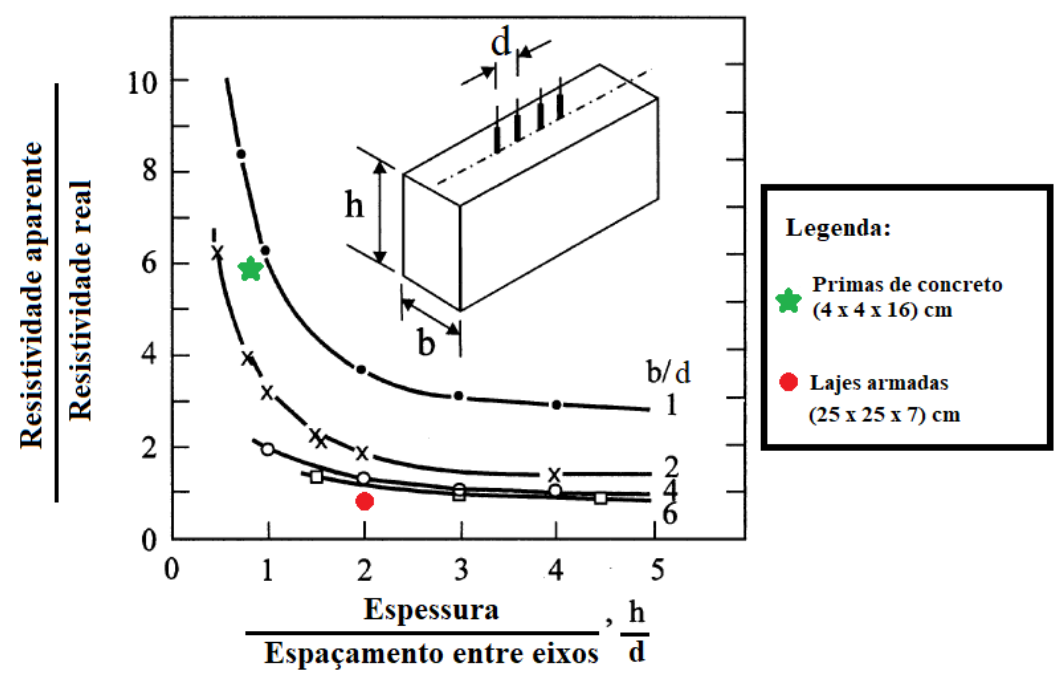

Figura 4: Efeito da geometria e dimensões de peças de concreto prismáticas na determinação da sua resistividade elétrica pelo método dos quatro eletrodos de Wenner. Adaptado de Gowers e Millard [23]

Os resultados da resistividade das amostras de concreto do presente estudo foram avaliados conforme a classificação de probabilidade de corrosão do CEB 192 [24] que é apresentada na Tabela 3

Tabela 3: Critério de avaliação da resistividade elétrica do concreto de acordo com o CEB 192 [24]

\begin{tabular}{cc}
\hline Resistividade elétrica com concreto $(\mathrm{K} \Omega . \mathrm{cm})$ & Indicação da probabilidade de corrosão \\
\hline 20 & Desprezível \\
10 a 20 & Baixa \\
5 a 10 & Alta \\
$\leq 5$ & Muito alta \\
\hline
\end{tabular}

Para corroborar os resultados e verificar a diferença e significância dos dados obtidos, foi realizado o teste Tukey com $95 \%$ de confiança.

\section{Resultados e discussões}

Os resultados dos ensaios mecânicos dos corpos de prova prismáticos são apresentados na Como já era esperado na avaliação dos parâmetros de resistência à compressão e à tração na flexão, não houve um grande aumento, $11 \%$ e 15\%, respectivamente, quando comparadas às idades de 28 dias e 100 dias (Figura 5). 0 aumento da resistência mecânica do presente estudo ficou próximo da descrita por 
GODINHO, J.P. et al., INFLUÊNCIA DO FORMATO DOS CORPOS DE PROVA E DA IDADE DE CURA NAS LEITURAS DE VELOCIDADE DE ULTRASSOM, RESISTIVIDADE ELÉTRICA E RESISTÊNCIA À COMPRESSÃO AXIAL. $3^{\circ}$ Simpósio Paranaense de Patologia das Construções (30 SPPC), artigo 3SPPC1011, pp. 116 - 129. 2018. DOI: 10.4322/2526-7248.011

Mehta e Monteiro [2], onde os autores afirmam que concretos sem adições minerais no cimento e com níveis de clínquer superiores a $90 \%$ (como no caso do presente trabalho) ganham aproximadamente $90 \%$ da resistência aos 28 dias de cura úmida.

Tabela 4 e na Figura 5.

Como já era esperado na avaliação dos parâmetros de resistência à compressão e à tração na flexão, não houve um grande aumento, 11\% e 15\%, respectivamente, quando comparadas às idades de 28 dias e 100 dias (Figura 5). O aumento da resistência mecânica do presente estudo ficou próximo da descrita por Mehta e Monteiro [2], onde os autores afirmam que concretos sem adições minerais no cimento e com níveis de clínquer superiores a $90 \%$ (como no caso do presente trabalho) ganham aproximadamente $90 \%$ da resistência aos 28 dias de cura úmida.

Tabela 4: Resultados dos ensaios mecânicos dos corpos de prova

\begin{tabular}{|c|c|c|c|c|c|}
\hline & \multicolumn{4}{|c|}{ Corpos de prova prismáticos } & \multirow{3}{*}{$\begin{array}{c}\text { Corpos de prova cilíndricos } \\
\text { Compressão axial (MPa) } \\
28 \text { dias }\end{array}$} \\
\hline & \multicolumn{2}{|c|}{$\begin{array}{c}\text { Compressão axial } \\
\text { (MPa) }\end{array}$} & \multicolumn{2}{|c|}{$\begin{array}{l}\text { Tração na flexão } \\
\text { (MPa) }\end{array}$} & \\
\hline & 28 dias & 100 dias & $\begin{array}{c}28 \\
\text { dias }\end{array}$ & 100 dias & \\
\hline $\mathrm{N}$ & 10 & 10 & 5 & 5 & 5 \\
\hline Média & 40,6 & 45,8 & 7,6 & 8,9 & 28,7 \\
\hline Desvio Padrão & 1,4 & 0,7 & 0,3 & 0,8 & 2,3 \\
\hline Coef.Var.(\%) & 3,5 & 1,5 & 4,1 & 9,2 & 8,1 \\
\hline Mínimo & 38,3 & 44,6 & 7,3 & 8,2 & 26,5 \\
\hline Máximo & 42,4 & 46,6 & 8,0 & 9,8 & 31,9 \\
\hline
\end{tabular}

Legenda: $\mathrm{N}$ - número de corpos de prova; Coef.Var - Coeficiente de variação

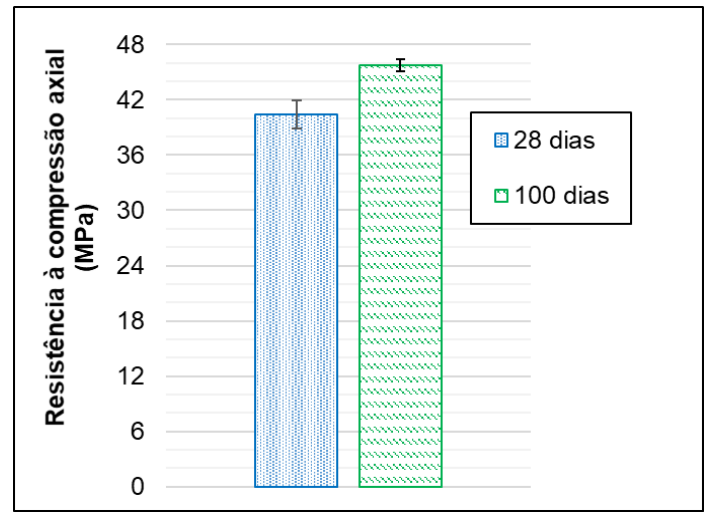

(a)

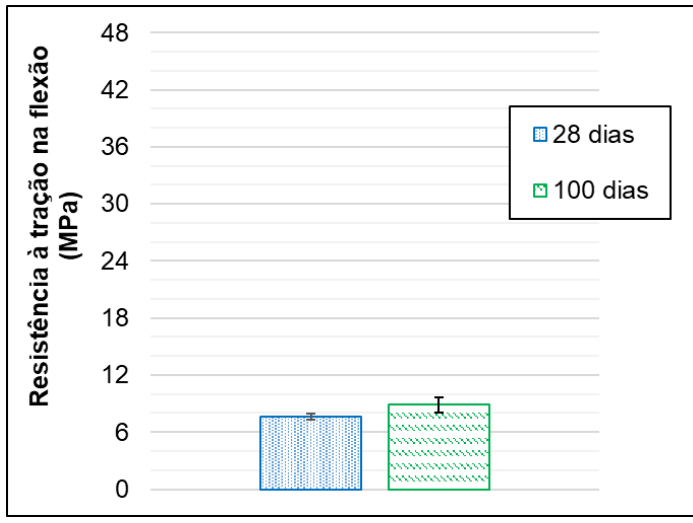

(b)

Figura 5: Ensaios mecânicos realizados nos corpos de prova prismáticos.

(a) resistência à compressão axial; (b) resistência à tração na flexão

Na Figura 6 é apresentada a resistência à compressão axial aos 28 dias, para os dois tipos de corpos de prova rompidos neste estudo.

Analisando a Figura 6 foi possível notar que a resistência à compressão dos corpos de prova prismáticos foi $29 \%$ superior aos dos corpos de prova cilíndricos, resultados estes que já eram esperados, visto que o fator de confinamento dos corpos de prova prismático é maior e resulta em uma maior restrição da prensa. 
Outro fator que justifica essa diferença de valores é a relação altura/diâmetro (h/d), sendo igual a 1 para os prismáticos e 2 para os cilíndricos.

Capraro et al. [25] evidenciaram a mesma tendência dos resultados apresentados na Figura 6 do presente trabalho, os autores avaliaram a influência da forma e dimensão de corpos de prova de argamassa nos resultados dos ensaios de resistência à compressão axial. Os autores observaram que, para todos os casos, argamassas de traços fortes e fracos, os resultados das configurações cilíndricas são menores quando comparados aos corpos de prova prismáticos, sendo que a redução da resistência à compressão dos corpos prova cilíndricos para os prismáticos foi de aproximadamente $42 \%$.

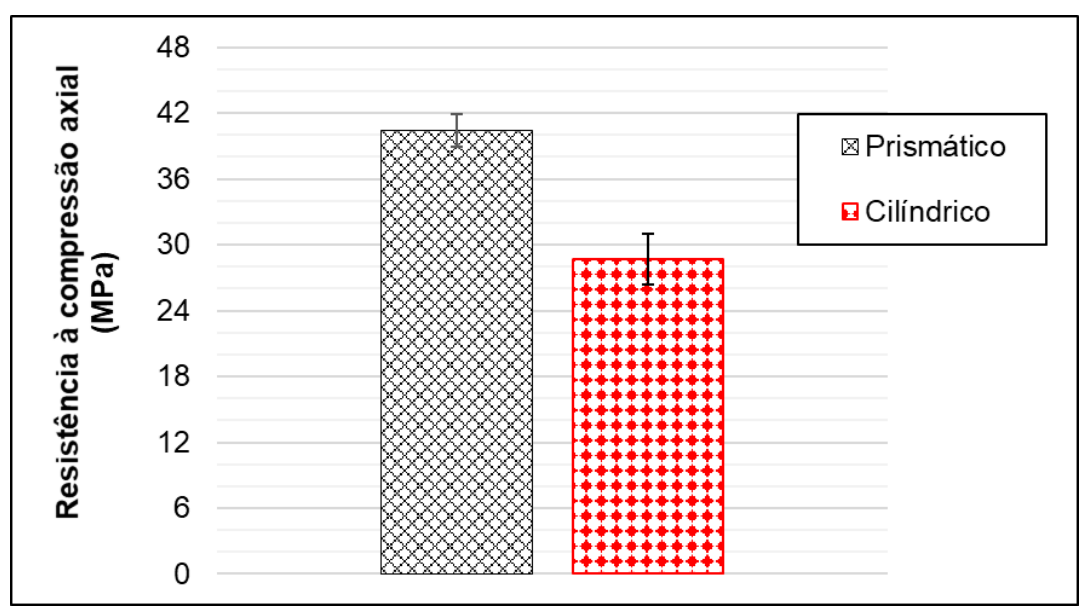

Figura 6: Resistência à compressão axial aos 28 dias nos corpos de prova de concreto (cilindros de $10 \mathrm{~cm} \times 20 \mathrm{~cm}$; prismas $4 \mathrm{~cm} \times 4 \mathrm{~cm} \times 16 \mathrm{~cm}$ )

Na Figura 7 é apresentada a análise gráfica estatística pelo teste Tukey, onde os intervalos de confiança representam a interação entre as duas amostras e caso haja a intersecção entre o intervalo do limite inferior e superior das amostras com o eixo vertical zero, pode-se afirmar que as diferenças entre elas não são significativas.
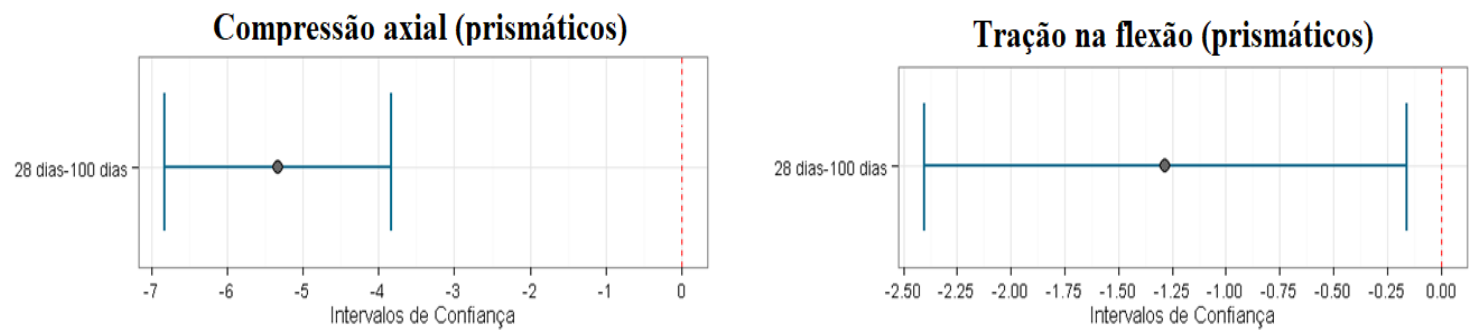

Resistência à compressão aos 28 dias

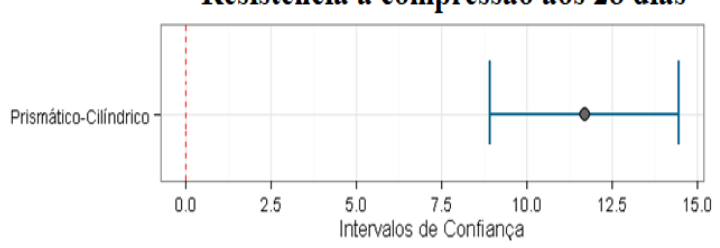

Figura 7: Teste Tukey para comparação múltipla de médias 
GODINHO, J.P. et al., INFLUÊNCIA DO FORMATO DOS CORPOS DE PROVA E DA IDADE DE CURA NAS LEITURAS DE VELOCIDADE DE ULTRASSOM, RESISTIVIDADE ELÉTRICA E RESISTÊNCIA À COMPRESSÃO AXIAL. $3^{\circ}$ Simpósio Paranaense de Patologia das Construções (30 SPPC), artigo 3SPPC1011, pp. 116 - 129. 2018. DOI: 10.4322/2526-7248.011

Aplicando o teste Tukey foi possível notar diferença estatística com significância superior a 95\% entre todos os ensaios mecânicos realizados.

Na Figura 8 é apresentada a variação média da velocidade de ultrassom para os três diferentes formatos de corpos de prova.

Para todos os formatos de corpos de prova, a velocidade de ultrassom aumentou ao longo do tempo. De acordo com Hernandez et al. [26] e Pereira e Medeiros [27], este é um indicativo de aumento de densidade (refinamento dos poros) e rigidez do concreto e, em consequência, também indica o aumento durabilidade do concreto. É possível notar que já na primeira leitura (aos 2 dias de cura) os corpos de prova prismáticos e as lajes armadas atingiram velocidades ultrassônicas maiores que $4.500 \mathrm{~m} / \mathrm{s}$, sendo, deste modo, considerados concretos de excelentes qualidades de acordo com a NBR 8802 [9].

$\mathrm{Na}$ análise com o teste Tukey, somente foi possível evidenciar diferença estatística dos corpos de prova cilíndricos, em relação aos outros dois formatos nos 2 dias e 16 dias de cura. De acordo com Naik e Malhorta [28] e Mehta e Monteiro [2], nas primeiras idades a hidratação do concreto é rápida e possui maior variação, podendo influenciar diretamente nas leituras de outros parâmetros como no caso da velocidade de ultrassom.

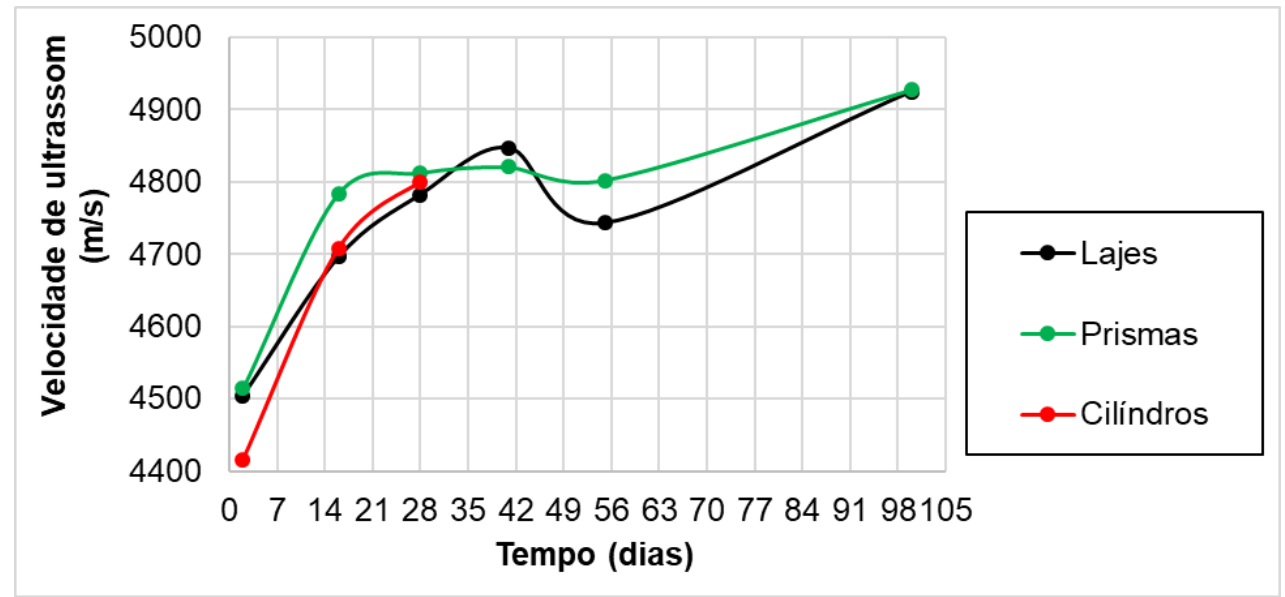

Figura 8: Velocidade de ultrassom para os três diferentes formatos de corpos de prova

São vários os fatores que influenciam nas leituras de velocidade de ultrassom do concreto, sendo eles: o tipo de cimento, grau de hidratação, umidade, condições de cura do concreto, relação água/cimento $(\mathrm{a} / \mathrm{c})$, dimensão do agregado entre outras $[9,28-30]$.

Aos 28 dias, 41 dias, 55 dias e 100 dias de cura, os formatos não tiveram diferença estatística significativas nas leituras e diante desses resultados, seriam necessárias leituras dos três diferentes formatos com idades mais avançadas, para poder evidenciar ou não com maior precisão, a influência do formato e dimensões dos corpos de prova nas leituras de velocidade de propagação de onda ultrassônica. 
Na Figura 9 é apresentada a resistividade elétrica para os três diferentes tipos de corpos de prova.

Analisando a Figura 9 e aplicando o teste de Tukey para os três formatos de corpos de prova, sem aplicação de fator de correção, foi possível notar diferença estatística nos resultados das leituras de resistividade elétrica do concreto, com exceção dos resultados aos dois dias de cura das lajes armadas em comparação aos cilindros sem fator de forma. Diante disto, é possível afirmar que as leituras de resistividade elétrica dependem diretamente das dimensões e formato dos corpos de prova, sendo necessário o cálculo de um fator de forma diferente para conversão a um meio semi-infinito, quando os corpos de prova têm uma relação b/d menor que 6 .

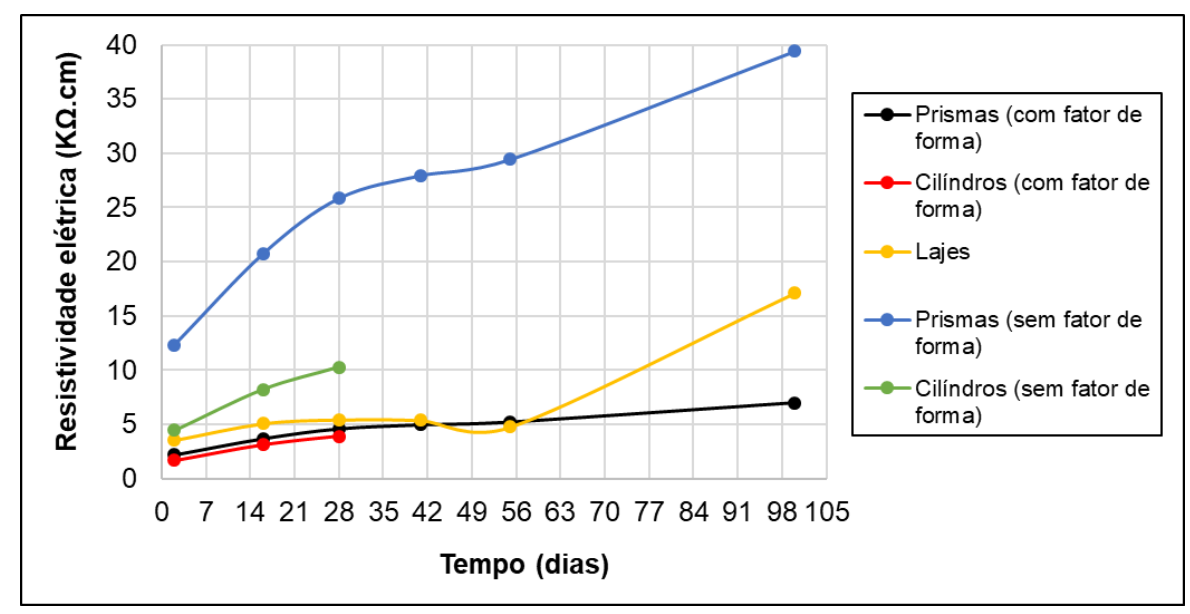

Figura 9: Resistividade elétrica para os três tipos de corpos de prova, antes e após a aplicação dos fatores de forma correspondentes

A resistividade elétrica para os três diferentes formatos de corpos de prova aumentou ao longo do tempo, comportamento também observado por Polder [31] e Medeiros-Junior et al. [32] e de acordo com esses autores os produtos da hidratação do concreto preenchem os poros e diminuem o tamanho e a ligação entre eles, dificultando assim a passagem de corrente e fazendo com que se aumentem os resultados de resistividade elétrica.

Neville [3] também afirma que este aumento da resistividade elétrica indica de forma indireta a diminuição dos poros e da sua interconectividade, em consequência o aumento de densidade, que contribui para dificultar a penetração de agentes agressivos para o interior do concreto, aumentando assim a sua durabilidade.

Após a aplicação do fator de forma, deve-se notar na Figura 9 que os resultados dos primas e cilindros ficaram na ordem de grandeza das leituras executadas nas lajes armadas até os 55 dias de cura, o que corrobora a teoria que as lajes armadas por terem uma relação b/d maior que 6 , não necessitam de aplicação de fator de forma, como no trabalho de Gowers e Millard [23].

Pode-se observar na Figura 9 que até aos 55 dias de cura os primas e os cilindros com aplicação do fator de forma e as lajes armadas sem aplicação do fator de forma, tiveram resultados estatisticamente equivalentes e de acordo com o CEB 192 
GODINHO, J.P. et al., INFLUÊNCIA DO FORMATO DOS CORPOS DE PROVA E DA IDADE DE CURA NAS LEITURAS DE VELOCIDADE DE ULTRASSOM, RESISTIVIDADE ELÉTRICA E RESISTÊNCIA À COMPRESSÃO AXIAL. $3^{\circ}$ Simpósio Paranaense de Patologia das Construções (30 SPPC), artigo 3SPPC1011, pp. 116 - 129. 2018. DOI: 10.4322/2526-7248.011

[24], que define os critérios de avaliação da resistividade elétrica, pode-se classificar o concreto destes três formatos como tendo uma probabilidade alta de corrosão.

Os resultados das leituras aos 100 dias para as lajes armadas tiveram um aumento de 3 vezes em relação aos primas e os cilíndricos na mesma idade, resultados estes que não eram esperados, pois segundo McCarter e Barclay [33] Gowers e Millard [23] e Medeiros [34] a presença de um elemento mais condutor (o aço carbono) na área de influência dos eletrodos do resistivímetro faz com que as leituras sejam subestimadas.

\section{Conclusões}

Diante dos resultados apresentados, pode-se concluir que:

- A forma e as dimensões dos corpos de prova influenciam diretamente nas leituras de resistência compressão axial, em decorrência do fator de confinamento e do fator de esbeltes que cada formato de corpo de prova possui.

- Nas primeiras idades de cura do concreto (2 dias e 28 dias), o formato dos corpos de prova tem influência nas leituras de velocidade de ultrassom, mas em idades superiores aos 28 dias de cura não foi possível evidenciar tal influência do formato e dimensões, desde que se utilize os corretos transdutores de diferentes frequências especificados pelo fabricante.

- As leituras de resistividade elétrica são influenciadas pelo formato e dimensões e desta forma, é necessário o cálculo de um fator de forma quando os corpos de prova têm uma relação b/d menor que 6.

\section{Agradecimentos}

Os autores expressam a sua gratidão as agências brasileiras CNPq, Capes e Fundação Araucária pelas bolsas e apoio financeiro, bem como a Universidade Federal do Paraná (UFPR) câmpus Curitiba, Centro Politécnico, ao Departamento de Construção Civil (DCC), ao Programa de Pós-Graduação em Engenharia de Construção Civil (PPGECC), ao Centro de Estudos de Engenharia Civil (CESEC), ao Laboratório de Materiais e Estruturas (LAME) e ao grupo de pesquisa de Patologia e Recuperação das Construções (PRC).

\section{Referências}

[1] Folić, R. (2009) Durability design of concrete structures - part 1: analysis fundamentals durability design of concrete structures. Architecture and Civil Engineering. vol. 7, no 1. p.1-18.

[2] Mehta, P. K.; Monteiro, P. J. (2014) Concreto: microestrutura, propriedades e materiais. 2a edição Brasileira. 4ํㅡㄹ Edição em inglês. São Paulo. Brasil.

[3] Neville, A. M. (2015) Propriedades do Concreto. 5a Ed. Bookman Editora.

[4] Monfore, G. E. (1968) The electrical resistivity of concrete. Journal of Portland Cement Association. no 224. p. 35-48. 
GODINHO, J.P. et al., INFLUÊNCIA DO FORMATO DOS CORPOS DE PROVA E DA IDADE DE CURA NAS LEITURAS DE VELOCIDADE DE ULTRASSOM, RESISTIVIDADE ELÉTRICA E RESISTÊNCIA À COMPRESSÃO AXIAL. $3^{\circ}$ Simpósio Paranaense de Patologia das Construções (30 SPPC), artigo 3SPPC1011, pp. 116 - 129. 2018. DOI: 10.4322/2526-7248.011

[5] Wilson, J. B. (1986) The electrical properties of concrete. Thesis submitted to the University of Edinburgh for the degree of doctor of philosophy. Department of Electrical Engineering. Scotland.

[6] Whittington, H. W.; Mccarter, J.; Forde, M. C. (1981) The conduction of electricity through concrete. Magazine of concrete research, v. 33, n. 114, p.48-60.

[7] Page, C. L.; Page, M. M. (Ed.). (2007) Durability of concrete and cement composites. Woodhead Publishing; 1ำ edition, (Elsevier), 416 p., july 14.

[8] Castellote, M.; Andrade, C.; Alonso, M. C. (2002) Standardization, to a reference of $25^{\circ} \mathrm{C}$, of electrical resistivity for mortars and concretes in saturated or isolated conditions. Materials Journal, v. 99, n. 2, p. 119-128.

[9] NBR 8802 (2013) Concreto endurecido - Determinação da velocidade de propagação de onda. Associação Brasileira de Normas Técnicas - ABNT, Rio de Janeiro, Brasil.

[10] Abdelouaheb, G.; Abdelhalim, B. (2016) Investigation of Concrete Segregation by Ultrasonic Pulse Velocity. Journal of Architectural Engineering Technology, v. 5, n. 3, p. 1-6.

[11]Ribeiro, D. V.; Sales, A.; Souza, C.; Almeida, F.; Cunha, M.; Lourenço, M.; Helene, P. (2014) Corrosão em estruturas de concreto armado: teoria, controle e métodos de análise. Editora Campus.

[12] NBR 6118 (2014) Projeto de Estruturas de Concreto - Procedimento. Associação Brasileira de Normas Técnicas - ABNT, Rio de Janeiro, Brasil.

[13] NBR 5738 (2003) Concreto - Procedimento para moldagem e cura de corpos de prova. Associação Brasileira de Normas Técnicas - ABNT, Rio de Janeiro, Brasil.

[14]NM 248 (2003) Agregados - Determinação da composição granulométrica. Asociación Mercosur de Normalización - AMN. São Paulo/SP, Brasil.

[15]NM 52 (2003) Agregado miúdo - Determinação da massa específica e massa específica aparente. Asociación Mercosur de Normalización - AMN. São Paulo/SP, Brasil.

[16]NM 53 (2009) Agregados graúdo - Determinação da massa específica aparente e absorção de água. Asociación Mercosur de Normalización - AMN. São Paulo/SP, Brasil.

[17]NBR 7215 (1996) Cimento Portland - Determinação da resistência à compressão. Associação Brasileira de Normas Técnicas - ABNT, Rio de Janeiro, Brasil.

[18]NBR 13279 (2005) Argamassa para assentamento e revestimento de paredes e tetos - Determinação da resistência à tração na flexão e à compressão. Associação Brasileira de Normas Técnicas - ABNT, Rio de Janeiro, Brasil.

[19]Proceq S. A (2017) Manual ultrassom Pundit Lab. Disponível em: https://www.proceq.com/uploads/tx_proceqproductcms. Acesso em 01 de abril de 2018. 
GODINHO, J.P. et al., INFLUÊNCIA DO FORMATO DOS CORPOS DE PROVA E DA IDADE DE CURA NAS LEITURAS DE VELOCIDADE DE ULTRASSOM, RESISTIVIDADE ELÉTRICA E RESISTÊNCIA À COMPRESSÃO AXIAL. $3^{\circ}$ Simpósio Paranaense de Patologia das Construções (30 SPPC), artigo 3SPPC1011, pp. 116 - 129. 2018. DOI: 10.4322/2526-7248.011

[20]UNE 83988-2 (2012) Durabilidad del hormigón - determinación de la resistividad - Parte 2: Método de las cuatro puntas o de Wenner. Asociación Española de Normalización y Certificación - AENOR, Madrid, España.

[21] Presuel-Moreno, F.; Liu, Y.; Wu, Y.-Y. (2013) Numerical modeling of the effects of rebar presence and/or multilayered concrete resistivity on the apparent resistivity measured via the Wenner method. Construction and Building Materials, vol. 48, pp. 16- 25 .

[22]Proceq S. A (2017) Manual Resipod. Disponível em: https://www.proceq.com/uploads/tx_proceqproductcms/import_data/files/Resipod_Sales \%20Flyer_Portuguese_high.pdf. Acesso em 01 de abril de 2018.

[23] Gowers, K. R.; Millard, S. G. (1999) Measurement of concrete resistivity for assessment of corrosion severity of steel using Wenner technique. ACI Materials Journal, v. 96, n. 5, p.536-541, september-october.

[24]CEB - COMITÉ EURO-INTERNACIONAL DU BÉTON. (1989) Diagnosis and assessment of concrete structures. State of the art report. Bulletin 192. Lausanne: CEB, $120 \mathrm{p}$.

[25] Capraro, D. F. A.; Capraro, A. P. B.; Argenta, M. A.; Medeiros, M. H. F. (2018, no prelo) Avaliação experimental e numérica da influência da forma e dimensão de corpos de prova de argamassa em ensaios de compressão. Revista IBRACON de Estruturas e Materiais, Instituto Brasileiro do Concreto.

[26]Hernández, M. G.; Izquierdo, M. A. G.; Ibáñez, A.; Anaya, J. J.; Ullate, L. G. (2000) Porosity estimation of concrete by ultrasonic NDE, Ultrasonics, v. 38, n. 1-8, p. 531-533.

[27]Pereira, E., Medeiros, M.H.F. (2012) Ensaio de "Pull Off" para avaliar a resistência à compressão do concreto: uma alternativa aos ensaios normalizados no Brasil. Revista IBRACON de Estruturas e Materiais, Instituto Brasileiro do Concreto, v. 5, n. 6, p.757-768.

[28]Naik, T. R., Malhorta, V. M. (1991) The ultrasonic pulse velocity method. In: Handbook on non-destructive testing of concrete. p. 169-188.

[29]Ferrari, V. J.; Padaratz, I. J. (2003) Aplicação de ondas ultra-sônicas na detecção das primeiras fissuras em vigas de concreto armado e na avaliação da resistência à compressão. Acta Scientiarum. Technology, v. 25, n. 2, p. 185191.

[30] Cosmes-López, M. F.; Castellanos, F.; Cano-Barrita, P. F. de J. (2017) Ultrasound frequency analysis for identification of aggregates and cement paste in concrete. Ultrasonics, v. 73, p. 88-95.

[31]Polder, R. B. (1995) Chloride diffusion and resistivity testing of five concrete mixes for marine environment. 1st RILEM workshop on Chloride Penetration into Concrete, 15-18 October 1995, St Rémy lès Chevreuse, France. 9 p.

[32]Medeiros-Junior, R. A.; Lima, M. G.; Medeiros, M. H. F.; Real, L. V. (2014) Investigação da resistência à compressão e da resistividade elétrica de 
GODINHO, J.P. et al., INFLUÊNCIA DO FORMATO DOS CORPOS DE PROVA E DA IDADE DE CURA NAS LEITURAS DE VELOCIDADE DE ULTRASSOM, RESISTIVIDADE ELÉTRICA E RESISTÊNCIA À COMPRESSÃO AXIAL. $3^{\circ}$ Simpósio Paranaense de Patologia das Construções (30 SPPC), artigo 3SPPC1011, pp. 116 - 129. 2018. DOI: 10.4322/2526-7248.011

concretos com diferentes tipos de cimento. Revista ALCONPAT, v. 4, p. 116128.

[33]McCarter, W. J. \& Barclay, S. A (1993) Comparison of two methods for resistivity measurements on repair mortar for cathodic protection systems, Revista: Cement and Concrete Research, vol. 23, pp. 1178-1184.

[34]Medeiros, M. H. F. (2001) Estudo de variáveis que influenciam nas medidas de resistividade de estruturas de concreto armado. Revista Engenharia Civil da Universidade do Minho, v.12, Guimarães, 2001. 